\title{
GENERATION OF HIGH POWER HEAVY ION BEAMS AT GSI
}

\author{
P. Spiller, K. Blasche, O. Boine-Frankenheim, M. Emmerling, \\ B. Franczak, I. Hofmann, S. Lund (*), U. Ratzinger \\ GSI - Gesellschaft für Schwerionenforschung, D-64220 Darmstadt, Germany \\ (*) LLNL - Lawrence Livermore National Laboratory, Livermore, CA94550, USA
}

\begin{abstract}
Besides laser- and light ion beams, short pulse duration bunches of intense heavy ion beams are attractive to deposit high specific power in experimental targets. Such volume heated matter is transformed into a plasma state that is characterized by its high density. The GSI accelerator facility offers a unique possibility for the generation of such a solid state density plasma. After the installation of the new high current injector, intense beams of low charge state uranium ions $\left(U^{28+}\right)$ will be available. To achieve high target power densities with these beams, a strong longitudinal compression has to be performed. For this purpose, a new type of $\mathrm{RF}$ compressor cavity has been developed for the heavy ion synchrotron SIS. In the framework of a machine development program, first tests of the required bunch manipulations have been done with the available SIS cavities.
\end{abstract}

\section{INTRODUCTION}

Short pulse duration ion bunches can easily be produced by means of a high revolution frequency, or equivalently, high beam energy. However, the most interesting beam energy for plasma physics experiments is defined by the ion range in the target and the focal spot radius. Thus, for solid state density targets, $100-300 \mathrm{MeV} / \mathrm{u}$ is an appropriate range of energy for heavy ions. However, at $200 \mathrm{MeV} / \mathrm{u}$ the revolution time in the SIS is about $1.3 \mu \mathrm{s}$, which is far too long for optimal target heating. Taking into account the hydrodynamic target response, the ion pulse length has to be shortened by a factor of 20 or more.

Furthermore, optimum plasma generation with the present range of achievable total beam energies requires a single bunch of ions. Due to an insufficiently, low minimum frequency of the SIS cavities, a minimum of harmonic 4 can be used for acceleration of $U^{28+}$. Thus, at least four ion bunches must be merged into one before final bunching is carried out with a new low-frequency cavity.

\section{RF-MANIPULATIONS AND LONGITUDINAL COMPRESSION}

In March 1997, the first attempt of single bunch generation in the SIS was carried out [1]. The method used is based on adiabatic debunching, followed by a rebunching on the first harmonic. In adiabatic compression the final bunch length $l_{f}$ is determined by : $l \propto V^{1 / 4}$. With a maximum available RF voltage of $V=14 \mathrm{kV}$ per cavity and a typical momen- tum spread of dp/p $= \pm 5 \cdot 10^{-4}$ a coasting beam of $A r^{11+}$ ions could be captured in a single bunch with a pulse duration of $550 \mathrm{~ns}$. No particle losses were observed at this process.

For the final compression of the single bunch, we consider a fast compression with a 90 degree rotation of the longitudinal phase space. This concept was tested in the Experimental Storage Ring ESR [2] and has been applied in experiments in the SIS since November 97 [3] [4]. The phase space rotation in fast compression is initiated by a fast jump of the RF-voltage amplitude.
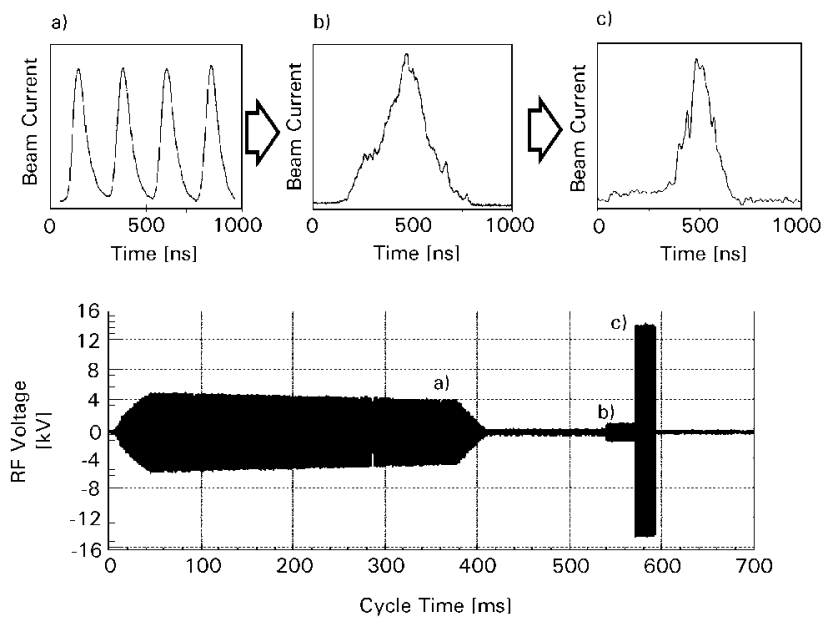

Figure 1: Measured bunch current signals at different stages of the acceleration cycle together with the measured RF gap voltage over the modified acceleration process. Shown is a) the typical four-bunch structure after acceleration with $\mathrm{h}=4, \mathrm{~b})$ a single bunch profile after adiabatic debunching and rebunching at $\mathrm{h}=1$ and $\mathrm{c}$ ) a single bunch after fast bunch compression.

The rise time of the voltage must be much shorter than the synchrotron period of longitudinal particle oscillations. The measured rise time of the SIS cavities is $150 \mu$ s which is sufficiently short relative to a typical synchrotron period of about $1 \mathrm{~ms}$. Measurements of the full cycle of acceleration, adiabatic debunching and rebunching, and final fast bunch rotation are illustrated in Fig. 1.

However, measurements have shown that phase stability is essential for a stable compression. Figure 2 shows the beam current signal in the SIS, measured with a capacitive pick-up probe, over several synchrotron periods after the 
RF voltage jump. Due to small RF phase instabilities coherent oscillations of the bunch are excited. The observed phase instability is due to a dependence of the ferrite permeability $\mu$ on the voltage amplitude. The varying permeability leads to a change of the eigenfrequency of the cavity. Although the RF phase is stabilized after the jump, these oscillations can be observed over many periods. These oscillations may dilute the longitudinal bunch emittance, limiting compression for a given final momentum spread.

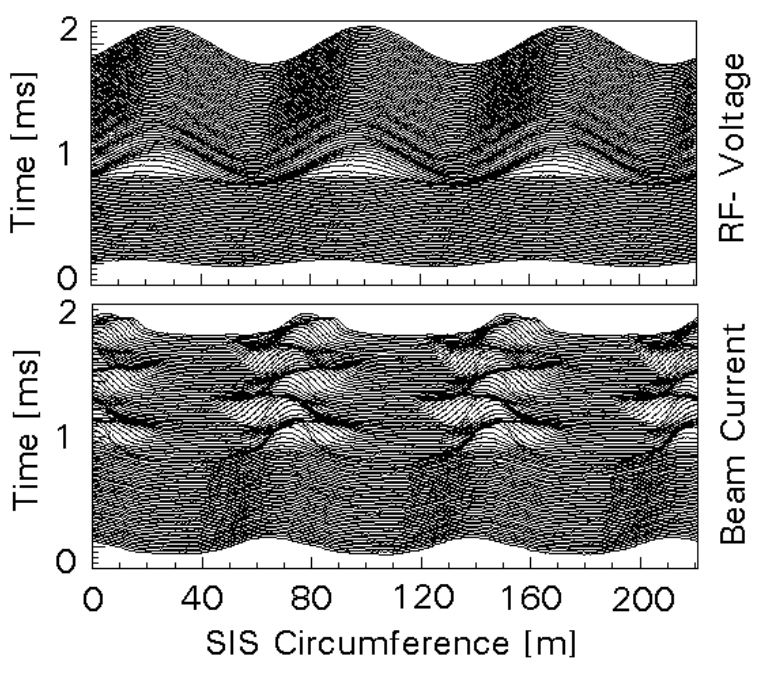

Figure 2: Evolution of the RF gap voltage and the beam current during fast compression. Strong coherent bunch oscillations are visible. These oscillations are excited by RF phase variations of about $15^{\circ}$ at the fast voltage jump.

The strongest compression factor can be achieved with rather small prebunching amplitudes, according to : $l_{f} \propto$ $\sqrt{A / q\left(V_{i} / V_{f}\right)} \cdot(d p / p)_{i}$. Here $\mathrm{A}$ is the mass number, $\mathrm{q}$ is the charge state and the subscripts $i$ and $f$ denote initial and final values. This equation assumes an initial stationary bunch, matched to the RF bucket and linear bunching. In case of smaller prebunching amplitudes, distortions of the longitudinal phase space occur due to the nonlinearity of the RF-voltage seen by the mismatched bunch.
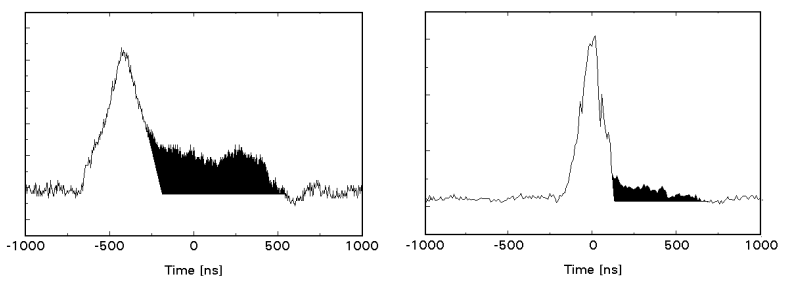

Figure 3: Bunch signal after extraction without (left) and with $1 \mathrm{kV}$ (right) prebunching. The shaded areas indicate the offset and tail depending on the amount of prebunching.

The nonlinear RF-voltage causes an S-shaped phase space wrapping with associated nonlinear pulse lengthening. In an experimental program using an $A r^{11+}$-beam the influence of the prebunching amplitude on the final longitudinal distribution was studied. Fig. 3 shows the longitudinal profile of an extracted bunch for no prebunching and with $1 \mathrm{kV}$ prebunching. A final pulse duration of $350 \mathrm{~ns}$ could be achieved. Table 1 shows results of 1D PIC simulations of a fast compression of a weak prebunched 200 $\mathrm{MeV} / \mathrm{u} U^{28+}$-beam. The fraction $N_{B} / N$ of particles captured in the RF bucket is calculated for different levels of prebunching. Furthermore, the mid-pulse current gain factor $I / I_{0}$ which indicates the peak power of the bunch is listed. The final RF amplitude was assumed to be $100 \mathrm{kV}$.

Table 1 : Current gain factor $I / I_{0}$, fraction of captured particles $N_{B} / N$, and final bunch lengths $z_{f}$ for different prebunching amplitudes.

\begin{tabular}{|c|c|c|c|c|c|}
\hline $\begin{array}{c}V_{i} \\
{[k V]}\end{array}$ & $\begin{array}{c}z_{f} \\
{[\mathrm{~m}]}\end{array}$ & $\begin{array}{c}\tau_{f} \\
{[\mathrm{~ns}]}\end{array}$ & $I / I_{0}$ & $N_{B} / N$ & $\begin{array}{c}(d p / p)_{f} \\
{[\%]}\end{array}$ \\
\hline \hline 0 & 7 & 36.7 & 10.7 & 0.46 & $\approx 0.5$ \\
\hline 1 & 10.5 & 55.1 & 12 & 0.78 & $\approx 0.5$ \\
\hline 2 & 11.5 & 60.3 & 11.5 & 0.82 & $\approx 0.5$ \\
\hline 3 & 12.0 & 63 & 11.4 & 0.84 & $\approx 0.5$ \\
\hline 5 & 14.0 & 73.5 & 10.7 & 0.92 & $\approx 0.5$ \\
\hline
\end{tabular}

For a fixed prebunching amplitude of $2 \mathrm{kV}$ the influence of beam loading on the cavity is studied for different impedances. Table 2 shows the degradation of the final beam parameters with increasing impedance.

Table 2 : The filamentation of the phase space by space charge interaction with the cavity impedance degrades the achievable peak current. For an assumed prebunching voltage of $2 \mathrm{kV}$ the same parameters as in Table 1 are listed.

\begin{tabular}{|c|c|c|c|c|c|}
\hline $\begin{array}{c}R_{s} \\
{[k \Omega]}\end{array}$ & $\begin{array}{c}z_{f} \\
{[\mathrm{~m}]}\end{array}$ & $\begin{array}{c}\tau_{f} \\
{[\mathrm{~ns}]}\end{array}$ & $I / I_{0}$ & $N_{B} / N$ & $\begin{array}{c}(d p / p)_{f} \\
{[\%]}\end{array}$ \\
\hline \hline 0 & 11.5 & 60.3 & 11.5 & 0.82 & $\approx 0.5$ \\
\hline 1 & 11.5 & 60.3 & 11.5 & 0.82 & $\approx 0.5$ \\
\hline 3 & 11.5 & 60.3 & 11.3 & 0.81 & $\approx 0.5$ \\
\hline 4 & 11.5 & 60.3 & 11 & 0.78 & $\approx 0.5$ \\
\hline 5 & 11.5 & 60.3 & 10.5 & 0.74 & $\approx 0.5$ \\
\hline 10 & 10 & 52 & 7 & 0.43 & $\approx 0.5$ \\
\hline
\end{tabular}

A significant drop of the peak current is visible starting from a total impedance of about $5 k \Omega$.

Further detailed simulations on the compression process in a dispersive ring lattice and the effect on the transverse emittance are presented in a corresponding paper [7].

\section{THE COMPRESSOR CAVITY STUDY}

The highest energy deposition in a target will be achieved with an intense $U^{28+}$-beam [5]. With the new high current injector [6], $2 \cdot 10^{11}$ uranium ions will be available at the end of 1999. Accelerated up to the maximum rigidity of 18 
Tm, a total beam energy of $2 \mathrm{~kJ}$ can be produced for experiments. When focused onto a small spot $(R \leq 1 \mathrm{~mm})$ on the target [8], a specific energy deposition of up to $50 \mathrm{~kJ} / \mathrm{g}$ is expected. At this level of energy deposition the characteristic heating time, which is determined by the sound velocity of the plasma, is about $50 \mathrm{~ns}$. A bunch with a matched pulse duration can only be generated with the installation of a dedicated bunch compressor cavity. Such cavities have been studied by the GSI RF group [9], using magnetic alloy technology. For compression of $100 \%$ of the beam particles down to a pulse duration of $50 \mathrm{~ns}$, a total RF-voltage of $200 \mathrm{kV}$ is required. This voltage must be produced at a fixed frequency of about $0.8 \mathrm{MHz}$ for less than $100 \mu \mathrm{s}(1 / 4$ of the synchrotron period).

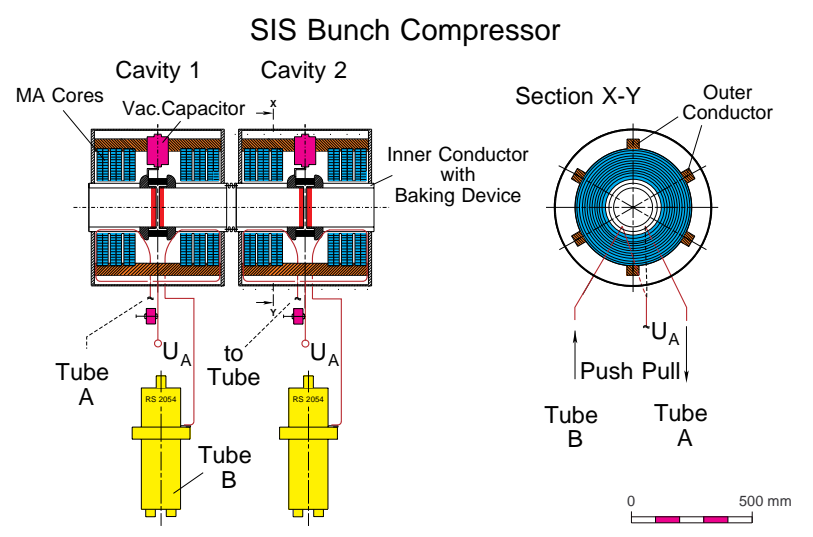

Figure 4: Schematic of a SIS bunch compressor cavity based on magnetic alloy cores. Shown are two out of six cavities. Each cavity is driven by a final stage in push-pull operation.

Table 3: Comparison of the technical parameters of the existing SIS ferrite loaded cavities and of the proposed MA compressor cavity.

\begin{tabular}{|l|c|c|}
\hline & SIS Cavity & Compressor \\
\hline Inductive Load & Ferrites & Magnetic Alloy \\
\hline $\begin{array}{l}\text { Frequency tuning } \\
\text { range [MHz] }\end{array}$ & $0.85-5.4$ & 0.8 \\
\hline Peak RF-voltage [kV] & 16 & 40 \\
\hline Length [m] & 2.9 & 0.7 \\
\hline Pulse duration $[\mu \mathrm{s}]$ & cont. & 100 \\
\hline Duty cycle [\%] & 100 & 0.01 \\
\hline Voltage rise time [ $\mu \mathrm{s}]$ & 150 & $\leq 30$ \\
\hline $\begin{array}{l}\text { Power dissipation - } \\
\text { Peak [kW] }\end{array}$ & 40 & 500 \\
\hline $\begin{array}{l}\text { Power dissipation } \\
\text { Mean [kW] }\end{array}$ & 40 & 0.05 \\
\hline Cooling of cores & water & air \\
\hline Gap impedance $[\mathrm{k} \Omega$ ] & 2.5 & 0.68 \\
\hline
\end{tabular}

The inductive load of the new cavity type consists of cores from amorphous metallic alloys (MA core). VITROVAC 6030F from Vacuumschmelze, is found to be an appropri- ate material. This material has a high saturation flux density of $0.82 \mathrm{~T}$ and the $\mu Q f$ product is constant over a wide range of RF-voltage. Therefore the observed phase instabilities at a fast voltage jump, as shown in figure 2, will not occur with the MA cavity. The MA material is available with a ribbon thickness of only $17 \mu \mathrm{m}$ and with a 2 $\mu \mathrm{m}$ thick interlaminar insulation. Proposed are 6 individual cavities, each capable of $40 \mathrm{kV}$ in an axial length of about $0.7 \mathrm{~m}$. Each cavity contains 2x 6 VITROVAC discs. Air cooling of the discs is sufficient because of the low duty factor of $0.01 \%$. The cavities are inductively coupled to the power amplifier by the anode supply cable guided around the MA cores (Fig. 4). In order to minimize the total impedance in the synchrotron, the gap will be shortcircuited when the cavity is not in operation. For the low duty cycle operation an adequate RF tube is the Siemens RS 2054. The peak power per cavity is on the order of 500 $\mathrm{kW}$. The gap impedances shown in table 3 are without fast feed back controls around the final amplifier. These controls are in the design stage.

With the high current injector and the new bunch compressor cavity we expect to achieve the beam and target parameters summarized in table 4.

Table 4: Beam and target parameters for the currently available RF-voltages and beam intensities of $A r^{11+}$-ion beams and future operation with $U^{28}$ ion beam.

\begin{tabular}{|c||c|c|}
\hline Ion species & $A r^{11+}$ & $U^{28+}$ \\
\hline$N_{B}$ & $2 \cdot 10^{10}$ & $2 \cdot 10^{11}$ \\
\hline$\epsilon_{i}[\mathrm{GeV}]$ & 12 & 47 \\
\hline$E_{0}[\mathrm{~kJ}]$ & 0.04 & 2 \\
\hline$\tau_{f}[\mathrm{~ns}]$ & 350 & 50 \\
\hline$P_{f}[\mathrm{GW}]$ & 0.11 & 40 \\
\hline$J_{s}\left[\mathrm{TW} / \mathrm{cm}^{2}\right]$ & 0.029 & 1.25 \\
\hline$J_{m}[\mathrm{TW} / \mathrm{g}]$ & 0.0014 & 0.5 \\
\hline$\rho \cdot l[\mathrm{~g} / \mathrm{cm}]$ & 13.04 & 1.5 \\
\hline$\epsilon_{m}[\mathrm{~kJ} / \mathrm{g}]$ & 0.47 & 26 \\
\hline
\end{tabular}

Acknowledgment : We thank P. Moritz, P. Kainberger and the plasma physics group for supporting the measurements.

\section{REFERENCES}

[1] GSI Scientific Report 1997, (1998)163

[2] G. Kalisch, GSI Report, GSI-94-05, (1993)

[3] S. Stöwe et.al., Nucl. Instr. and Meth. in Phys. Res. A (1998)61-67

[4] K. Blasche et.al., Proceedings of the $6^{\text {th }}$ EPAC, (1998) 1347

[5] R.W. Müller and P. Spiller, GSI Report, GSI-96-07,(1996)

[6] U. Ratzinger, Proc. of the 1996 Linac Conf.,Geneva,Vol.1 (1996) 288

[7] S.M. Lund, et. al. these proceedings

[8] M. Stetter et. al., Il Nuovo Cimento, Vol.106 A, N. 11(1725)

[9] M. Emmerling et. al. GSI Internal Report Nr. SIS29019STR 